\title{
The structure of the $\mathrm{Mn}_{4} \mathrm{Ca}^{2+}$ cluster of photosystem II and its protein environment as revealed by X-ray crystallography
}

\author{
James Barber* and James W. Murray \\ Division of Molecular Biosciences, Imperial College London, London SW7 2AZ, UK
}

\begin{abstract}
The location, structure and protein environment of the $\mathrm{Mn}_{4} \mathrm{Ca}^{2+}$ cluster, which catalyses the lightdriven, water-splitting reaction of photosystem II, has been revealed by X-ray crystallography. However, owing to the low resolutions of the crystal structures reported to date, and the possibility of radiation damage at the catalytic centre, the precise position of each metal ion remains unknown. To some extent, these problems have been overcome by applying spectroscopic techniques like extended $\mathrm{X}$-ray absorption fine structure. Taking into account the most recent results obtained with these two X-ray-based techniques, we have attempted to refine models of the structure of the $\mathrm{Mn}_{4} \mathrm{Ca}^{2+}$ cluster and its protein environment.
\end{abstract}

Keywords: photosystem II; structure; X-ray diffraction; manganese cluster; models; oxygen-evolving complex

\section{INTRODUCTION}

Photosystem II (PSII) uses sunlight to split water into its elemental constituents: dioxygen is released providing our planet with an aerobic atmosphere and a protective ozone layer, while the 'hydrogen' supplies reducing equivalents which, with additional input of light energy absorbed by photosystem I, are used to convert carbon dioxide into the organic molecules that constitute the biosphere. It is estimated that on an annual basis, PSII produces approximately $260 \mathrm{Gt}$ of dioxygen (Archer \& Barber 2004). The enormity of this reaction is remarkable given that water is a very stable compound and its oxidation is thermodynamically demanding. In total, it takes approximately $4 \mathrm{~V}$ of potential energy to generate a dioxygen molecule from two water molecules. In the case of PSII, this energy originates from the absorption of four photons of visible light $(h v)$,

$$
2 \mathrm{H}_{2} \mathrm{O} \stackrel{4 h v}{\longrightarrow} \mathrm{O}_{2}+4 e+4 \mathrm{H}^{+} \text {. }
$$

For this reason, the catalytic cycle of water oxidation relies on the storage of four oxidizing equivalents at an oxygen-evolving centre (OEC) within PSII, containing four $\mathrm{Mn}$ ions and $\mathrm{Ca}^{2+}$ and involves at least five chemical intermediates $\left(\mathrm{S}_{0}-\mathrm{S}_{4}\right)$ of the S-state cycle (reviewed in Diner \& Babcock (1996)). It remains one of the greatest challenges of photosynthesis research, and science in general, to elucidate the chemistry of the water-splitting reaction of PSII. Although a host of biophysical, biochemical and molecular biological

\footnotetext{
*Author for correspondence (j.barber@imperial.ac.uk).
}

Electronic supplementary material is available at http://dx.doi.org/10. 1098/rstb.2007.2208 or via http://journals.royalsociety.org.

One contribution of 20 to a Discussion Meeting Issue 'Revealing how nature uses sunlight to split water'. studies have been carried out to unravel the molecular mechanisms of this unique chemistry (recently reviewed in the various chapters of Wydrzynski \& Satoh (2005)), progress has been hampered until recently by the lack of a structure for the $\mathrm{Mn}_{4} \mathrm{Ca}^{2+}$ catalytic centre and details of its protein environment.

\section{STRUCTURES OF THE $\mathrm{Mn}_{4}$ CLUSTER WITHOUT $\mathrm{Ca}^{2+}$}

Although there had been considerable progress in determining the three-dimensional structure of PSII using electron microscopy, both electron crystallography and single particle analyses (reviewed in Barber $(2002,2003))$, the spatial resolutions obtained by these techniques were not sufficient to identify the electron density of the $\mathrm{Mn}_{4} \mathrm{Ca}^{2+}$ cluster. This situation was rectified by subjecting threedimensional crystals of PSII, isolated from the cyanobacterium Thermosynechococcus elongatus, to X-ray diffraction analysis (Zouni et al. 2001). This analysis generated an incomplete model of PSII at a resolution of $3.8 \AA$ : the tracing of C-alpha backbones of some subunits was not complete and there was no assignment of amino acids other than a tentative identification of D1-Tyr161 ( $\mathrm{Tyr}_{\mathrm{Z}}$ ) and D2-Tyr160 $\left(\operatorname{Tyr}_{\mathrm{D}}\right)$. However, this work did provide information on the positioning of cofactors involved in excitation energy transfer and charge separation. Most importantly, the analysis of the diffraction data revealed the first direct hints of the position of the $\mathrm{Mn}_{4}$ cluster within PSII. The electron density assigned to four $\mathrm{Mn}$ ions was checked by collecting anomalous diffraction data close to the Mn-absorption edge at $1.894 \AA$, and was found to be towards the lumenal surface of PSII on the D1 side of the pseudo-twofold axis, which relates the transmembrane helices of the D1- and D2-reaction centre proteins and the cofactors they bind. In this way, 
Zouni et al. (2001) confirmed the expectation that the location of the Mn cluster breaks the pseudo-twofold symmetry of the PSII reaction centre, and therefore placed it in the vicinity of the redox active Tyr161 of the D1 protein $(\mathrm{Yz})$ and close to the surface helix located in the loop joining the lumenal ends of the C- and D-transmembrane helix of the D1 protein (CD-helix). The electron density had a 'pear shape' when contoured at 5 sigma having dimensions of $6.8 \AA \times$ $4.9 \AA \times 3.3 \AA$ with the long axis approximately parallel to the CD helix and tilted at approximately $23^{\circ}$ to the membrane plane. Owing to the low resolution and incompleteness of the model, including the absence of side-chain positioning, the assignment of the four $\mathrm{Mn}$ ions within the density was rather arbitrary. A $\mathrm{Mn}$ ion was positioned in the three bulges of the 'pear-shaped' density to form an isosceles triangle with a fourth $\mathrm{Mn}$ ion placed in the centre of the triangle. The distance between the $\mathrm{Mn}$ ions was approximately $3 \AA$ and therefore approximately consistent with analyses by extended X-ray absorption fine structure (EXAFS; see Yachandra 2002). However, these authors did not propose a position for $\mathrm{Ca}^{2+}$ and did not model bridging ligands for the $\mathrm{Mn}$ ions.

The 3+1 organization of the four $\mathrm{Mn}$ ions modelled by Zouni et al. (2001) gave support to the arrangement previously suggested by Peloquin et al. (2000) and earlier by Hasegawa et al. (1999), and was also a feature of the crystallographic model of the Mn cluster derived by Kamiya \& Shen (2003). Using PSII isolated from Thermosynechococcus vulcanus, a cyanobacterium closely related to $T$. elongatus, these authors obtained a crystal structure at $3.7 \AA$ and provided additional information to that revealed by Zouni et al. (2001). The tracing of main chains was more complete and there was some effort made to assign amino acids, particularly those of the D1 and D2 proteins as well as to some regions of the chlorophyll-binding proteins, CP43 and CP47. The positioning of cofactors was essentially the same as that reported by Zouni et al. (2001), but Kamiya \& Shen also assigned density for two closely located carotenoid molecules on the D2 side of the reaction centre. As found by Zouni et al. (2001), the shape of the electron density for the Mn cluster was 'pear-like' when contoured at 5 sigma, indicative of the $3+1$ organization. However, Kamiya \& Shen modelled the four $\mathrm{Mn}$ ions so that they were approximately in the same plane, whereas Zouni et al. (2001) had placed the central $\mathrm{Mn}$ ion protruding towards the lumenal surface. Importantly, Kamiya \& Shen's map contained electron density connecting to that of the Mn cluster that was tentatively assigned to side chains of the D1 protein, including Ala344, Asp170 and Glu333 (or His332). They also suggested that, based on weak electron density, D1-His337, D1-Asp189 (or D1-His190) and D1-Tyr73 may also be coordinated to $\mathrm{Mn}$ ions of the cluster. Mutagenesis studies already supported the idea that several of these residues could be ligands for the Mn ions (Debus 2001; Diner 2001), including those located in the C-terminal domain of the D1 protein (Diner et al. 1991). As in the case of the earlier crystal structure (Zouni et al. 2001), the model of Kamiya \& Shen did not include a $\mathrm{Ca}^{2+}$ that was bound close to the $\mathrm{Mn}$ cluster.

\section{STRUCTURE OF THE $\mathrm{Mn}_{4}$ CLUSTER WITH $\mathrm{Ca}^{2+}$}

The first complete and refined structure of PSII came from the work of Ferreira et al. (2004), where over 5000 amino acids were assigned in the dimeric complex. Consequently, this $3.5 \AA$ model identified and gave the structures of 19 different subunits of the T. elongatus PSII complex (except for one low molecular weight intrinsic subunit tentatively assigned to PsbN). Moreover, it provided the first reliable information about the protein environments of all the major cofactors of PSII, not only that of the $\mathrm{Mn}_{4} \mathrm{Ca}^{2+}$ cluster but also for those involved in energy capture, excitation transfer and charge separation. Anomalous diffraction was collected at a wavelength of $1.89 \AA$ to obtain an electron density profile for the $\mathrm{Mn}$ ions and at $2.25 \AA$ to locate $\mathrm{Ca}^{2+}$ (at $2.25 \AA$, calcium has an anomalous signal which is approx. four times stronger than manganese). Again, the electron density attributed to the four $\mathrm{Mn}$ ions was pear-shaped, indicative of the $3+1$ organization. However, it is difficult to make a direct comparison with the corresponding density reported in the earlier crystal structures due to the unavailability of primary data. The Mn-anomalous difference map of Ferreira et al. (2004) correlated with one metal in the small domain and three in the large globular domain, whereas the $2.25 \AA$ wavelength map covers one metal ion in the large domain. In this way, three $\mathrm{Mn}$ ions and the $\mathrm{Ca}^{2+}$ were modelled as a trigonal pyramid with the $\mathrm{Ca}^{2+}$ at its apex, all located in the large domain. The fourth $\mathrm{Mn}$ ion was placed in the small domain.

The much improved quality of the electron density map led to the assignment of virtually all the amino acids in the PSII complex and, in particular, those in close proximity to the $\mathrm{Mn}_{4} \mathrm{Ca}^{2+}$ cluster. The positioning of side chains, the analyses of the anomalous diffraction data and the recognition that the ions were likely to be bridged by oxo bonds at distances suggested by EXAFS resulted in a detailed model for the $\mathrm{Mn}_{4} \mathrm{Ca}^{2+}$ cluster. The working model proposed by Ferreira et al. (2004; also see Barber et al. 2004) consisted of the three Mn ions (Mn1, Mn2 and Mn3) forming a cubane-like arrangement with the $\mathrm{Ca}^{2+}$ linked by oxo bonds. The fourth $\mathrm{Mn}$ (Mn4, the 'dangler' of Peloquin et al. (2000) was suggested to be linked to the $\mathrm{Mn}_{3} \mathrm{Ca}^{2+} \mathrm{O}_{4}$ cubane via the oxygen of one of its oxo bonds. At the resolution of the diffraction data, the precise positioning of the metal ions could not be determined. Therefore, for simplicity, the distances between the $\mathrm{Mn}$ ions in the cubane were taken as $2.7 \AA$ with the $\mathrm{Mn}-\mathrm{Ca}^{2+}$ distance in the cubane modelled at 3.4 ̊. The dangler $\mathrm{Mn}$ was positioned $3.3 \AA$ from the closest $\mathrm{Mn}$ ions of the cubane and approximately $4 \AA$ from the $\mathrm{Ca}^{2+}$. As a consequence, the model was based on three $\mathrm{Mn}-\mathrm{di}-\mu$-oxo-Mn bonds at $2.7 \AA$, three $\mathrm{Mn}$-di- $\mu$-oxo- $\mathrm{Ca}^{2+}$ bonds at $3.4 \AA$ and two Mn-mono- $\mu$-oxo-Mn bonds at $3.3 \AA$. Although the distances chosen were compatible with distances derived from EXAFS for the PSII metal cluster (Yachandra 2002), the predicted number of each type of bond was not. Nevertheless, density function calculations (Lundberg \& Siegbahn 2004; Sproviero et al. 2006) and chemical synthesis of a mixed $\mathrm{Mn}-\mathrm{Ca}$ complex (Misra et al. 2005) suggested that such an organization was chemically feasible despite there 
being no known similar structure in biology. Moreover, the model has provided an important basis for developing detailed mechanisms for the water-splitting reaction leading to dioxygen formation (McEvoy \& Brudvig 2004, 2006; Siegbahn \& Lundberg 2005; Siegbahn 2006).

Based on this organization of the metal ions, the $\mathrm{Mn}_{3} \mathrm{Ca}^{2+} \mathrm{O}_{4}$ cubane had four protein side chains as ligands: D1-Asp342 for Mn1; D1-Glu189 and D1His332 for Mn2; and CP43-Glu354 for Mn3 (possibly bidentate). The identification of the glutamate of CP43 as a $\mathrm{Mn}$ ligand was a surprise and is a residue of a conserved motif Gly-Gly-Glu-Thr-Met-Arg-Phe-TrpAsp, which forms a 310 helix in the large extrinsic loop joining the lumenal ends of transmembrane helices $\mathrm{V}$ and V1 of this protein. In addition to these four apparent protein ligands, it was noted that the C-terminal residue of the D1 protein, D1-Ala344, is located close to $\mathrm{Ca}^{2+}$ and that D1-His337 could be hydrogen bonded to one of the bridging oxo bonds of the cubane. Two side-chain densities were available as ligands for the dangler Mn4 outside the cubane cluster. These were identified as D1-Asp170 and D1-Glu333, and also noted was that D1-Asp61 might function as a ligand via a bridging water molecule. Because the coordination number is usually 6 or 7 for $\mathrm{Ca}^{2+}$ and 5 or 6 for $\mathrm{Mn}$, then according to the Ferreira et al. model there must be additional non-protein ligands such as water molecules or hydroxides. Nevertheless, Ferreira et al. (2004) did emphasize that there was non-protein electron density in the vicinity of $\mathrm{Mn}(4)$ and $\mathrm{Ca}^{2+}$, which they tentatively assigned to a carbonate ion that formed bridging ligands between the two metals. Moreover, the positioning of this carbonate ion adjacent to the redox active D1-Tyr161 $\left(\operatorname{Tyr}_{Z}\right)$ suggested that this could be the site for binding of two substrate water molecules and formation of dioxygen. Also located in this potential catalytic site are D1-Gln 165 and CP43-Arg357, which may provide hydrogen-bonding networks for deprotonation of the substrate water molecules during the catalytic cycle, while D1-Asp61 is strategically located at the mouth of a polar channel, which probably functions to facilitate the exit of protons to the lumenal surface. This channel is approximately $30 \AA$ long and composed of side chains of the D1 protein (Asp61 and Glu65), D2 protein (Lys317 and Glu312) and the extrinsic PsbO protein (Asp158, Asp222, Asp223, Asp224, His228 and Glu114). As well as acting as a pathway for removing protons, it probably also provides a route for supplying water molecules to the active site where both functions are aided by a $\mathrm{Ca}^{2+}$ bound at the lumenal end of the channel ligated by PsbO residues, Glu114, His231 and Glu54 (Murray \& Barber 2006). Recently, a closer analysis of the Ferreira et al. structure (Murray \& Barber 2007) indicated two additional channels leading from the OEC to the lumenal surface. One sufficiently polar so that it could also act as a $\mathrm{H}^{+}$/water channel while the less polar nature of the other suggests that it may promote rapid oxygen diffusion from the catalytic site.

The work of Ferreira et al. (2004) also established that D1-His 190 was in hydrogen-bonding distance to D1-Tyr161 as required, and predicted, for the oxidation of the latter by the primary electron donor of
PSII, P680, to generate the neutral tyrosine radical (Hoganson \& Babcock 1997).

The model proposed by Ferreira et al. (2004) has recently been analysed in considerable depth using density function quantum mechanics/molecular mechanics (QM/MM) analysis (Sproviero et al. 2006, 2007). The calculations assumed that the tentatively identified carbonate in the X-ray structure is replaced by a chloride ion in the active $S_{1}$ state of the watersplitting catalytic cycle, and that the assigned protein ligands were complemented by water and hydroxyl ligands to satisfy the coordination requirements of the five metal ions. Despite these adjustments, the calculated model for the metal cluster was remarkably similar to that proposed by Ferreira et al. (2004) and confirmed that it is a chemically stable structure even in the absence of protein ligands. Simulation of EXAFS spectra from the QM/MM model was also consistent with the experimental spectra obtained from nonorientated samples.

Towards the end of 2005, a new crystal structure of PSII isolated from T. elongatus was published by Loll et al. (2005), following on from an earlier study (Biesiadka et al. 2004). The resolution of the structure was reported to be $3.0 \AA$. In most part, this new model confirmed the subunit and amino acid assignment and side-chain positioning reported by Ferreira et al. (2004) with minor differences within the limitation imposed by the intermediate resolution of both structures. Nevertheless, the improved electron density map allowed Loll et al. to correct and extend the original assignment of carotenoid molecules by Ferreira et al. (2004) and to locate several bound lipid molecules. As in the earlier papers from this group (Zouni et al. 2001), the pearshaped electron density attributed to the metal cluster of the water-splitting site was interpreted as four Mn cations organized in a Y-shape or $3+1$ arrangement. Based on anomalous diffraction, the $\mathrm{Ca}^{2+}$ was placed in a position similar to that proposed by Ferreira et al. (2004), and recently confirmed by Kargul et al. (2007), whereas the positioning of three of the $\mathrm{Mn}$ ions were different from that of Ferreira et al. (2004) but less so from that of the Zouni et al. (2001) model. The fourth $\mathrm{Mn}$ ion was assigned to the narrow end of the pearshaped density in a slightly different position from that of the Zouni et al. (2001) or Ferreira et al. (2004) model. The Loll et al. model for the metal cluster, like that of Ferreira et al. (2001), had distances between the metal ions which were in line with those derived from EXAFS measurements (Yachandra 2002). They modelled di- $\mu$-oxo bridges of $2.7 \AA$ between Mn 1 and Mn2 and between $\mathrm{Mn} 2$ and Mn3, while Mn1-Mn3 and Mn3-Mn4 were modelled at $3.3 \AA$, as expected for mono- $\mu$-oxo bridging.

The electron density surrounding that due to the metals was assigned to the same amino acids first identified in the crystal structure of Ferreira et al. (2004), although there were differences in the precise location in some cases. Within the errors of the two models, Loll et al. confirmed the Ferreira et al. assignment of most of the key residues in the water-splitting site, such as D1-Tyr161, D1-His 190, D1-Glu165, D1-Asp62, D1-His337, CP43-Arg357 and the probable metal ligands D1-His332, D1-Glu333, D1-Glu189 

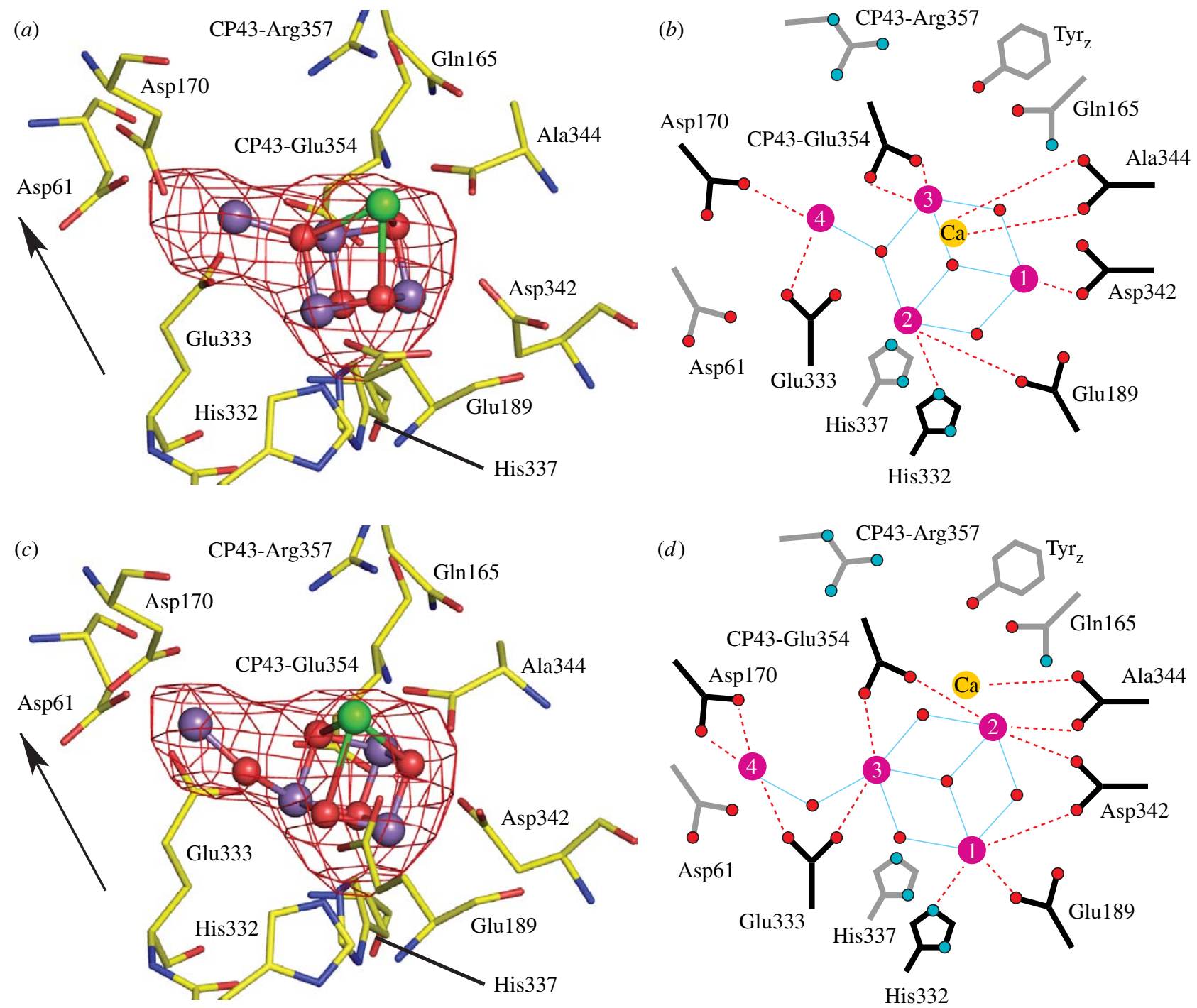

Figure 1. $(a, b)$ The water-splitting site as reported by Ferreira et al. (2004). (a) The $\mathrm{Mn}_{4} \mathrm{Ca}^{2+}$ cluster positioned within the Mn-anomalous difference map with amino acid side chains. (b) Schematic of the amino acid ligation pattern for the model in (a) with distance less than $2.8 \AA$ shown by connecting lines. $(c, d)$ Remodelling the water-splitting site using the native electron density maps of Ferreira et al. (2004) and Loll et al. (2005) and Mn-anomalous difference map of Ferreira et al. (2004), keeping the $\mathrm{Mn}_{3} \mathrm{Ca}^{2+} \mathrm{O}_{4}$ cubane of Ferreira et al. but with $\mathrm{Mn}_{4}$ linked to it via a single $3.3 \AA$ mono- $\mu$-oxo bridge. (c) Structure of the water-splitting site assuming a single mono- $\mu$-oxo bridge between $\mathrm{Mn}_{4}$ (dangler $\mathrm{Mn}$ ) and $\mathrm{Mn}_{3}$ of the $\mathrm{Mn}_{3} \mathrm{Ca}^{2+} \mathrm{O}_{4}$ cubane fitted into the $\mathrm{Mn}$-anomalous difference map by real-space refinement using the molecular graphics program, Coot (Emsley \& Cowtan 2004). The amino acid assignments and the positioning of their side chains are based on the native electron density maps of Ferreira et al. (2004) and Loll et al. (2005; see text). (d) Schematic of the amino acid ligation pattern for the model in (c) with distance less than $2.8 \AA$ shown by connecting lines. The Mn-anomalous difference map is shown in red and contoured at 5 sigma. The arrow indicates the direction of the normal to the membrane plane.

and CP43-Glu354. The most striking difference was the position of D1-Asp170 and to a lesser extent D1-Asp342 and D1-Ala344. With these differences, coupled with the different positioning of the $\mathrm{Mn}$ ions, Loll et al. (2005) proposed an amino acid coordination arrangement somewhat modified from that provided earlier by Ferreira et al. (2004). However, Loll et al. emphasized that their model for the water-splitting site was tentative, in part owing to the low resolution of the electron density map and in part owing to radiation damage. Working with the EXAFS group at Berkeley, and prior to the publication of the Loll et al. crystal structure of 2005, the same group had shown that doses of X-rays typical of those used for diffraction analyses caused the reduction of the $\mathrm{Mn}$ cluster and its associated structural changes (Yano et al. 2005). This problem of radiation damage occurring at the $\mathrm{Mn}$ cluster had also been highlighted by Dau et al. (2004) and investigated further by Grabolle et al. (2006).

Despite the uncertainties of the Ferreira et al. and Loll et al. models of the Mn cluster, the concept of bidentate bridging ligands proposed in the latter is worthy of serious consideration, given that this type of ligation is common in Mn-containing enzymes such as arginase (Kanyo et al. 1996), Escherichia coli ribonucleotide reductase (e.g. Sommerhalter et al. 2005), etc. (see Dismukes 1996) as well as in Mn-containing model compounds (Christou 1989). The presence of bridging carboxylate ligands may explain mutational studies, where the replacement of some of the proposed ligands with non-ligating side chains does not disrupt the cluster and inhibits water oxidation (Debus 2001; Diner 2001). 


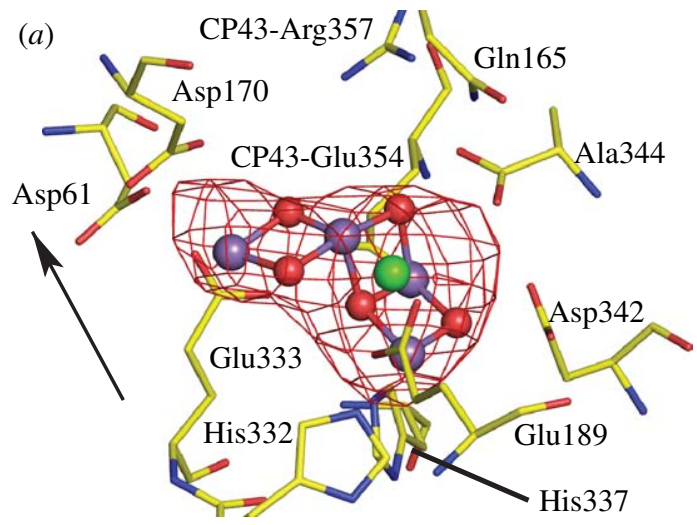

(b)

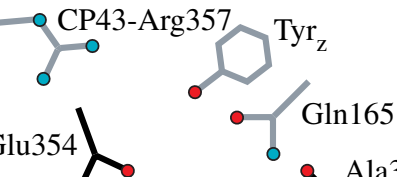

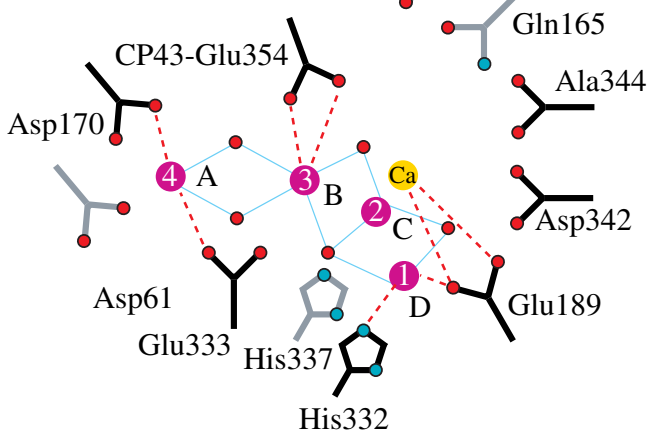

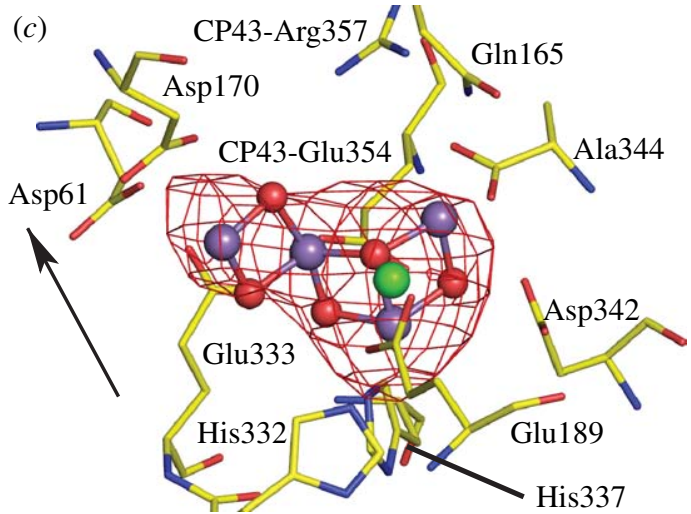

$(d)$

His 332
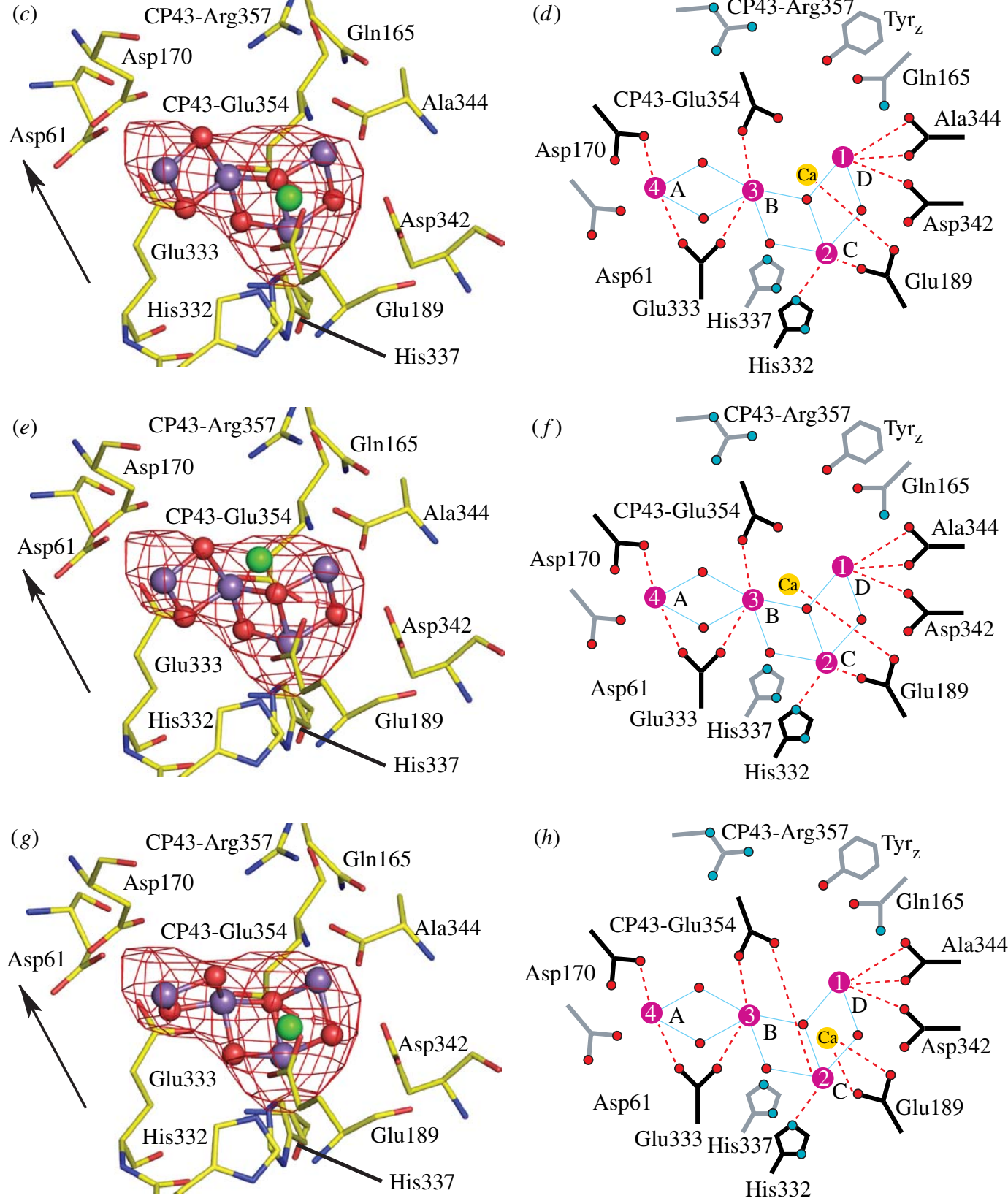

Figure 2. A refinement of the positioning of the EXAFS models (models I, II, IIa and III) of Yano et al. (2006) into the Mn-anomalous diffraction data of Ferreira et al. (2004), maintaining the side-chain positioning given in figure $1 c$. Each EXAFS model was fitted by real-space refinement against the Mn-anomalous difference map of Ferreira et al. (2004) using the molecular graphics program, Соот (Emsley \& Cowtan 2004). The Mn-anomalous difference map is shown in red and contoured at 5 sigma. $a, c, e$ and $g$ are for EXAFS models I, II, IIa and III, respectively, while $b, d, f$ and $h$ are the schematic for each showing potential ligation patterns where the lines represent distances of $2.8 \AA$ or less. The arrows indicate the direction of the normal to the membrane plane. 


\section{REINTERPRETATIONS OF THE OEC STRUCTURE}

In this section, we have undertaken a reinterpretation of the structure of the OEC using currently available information and favouring bidentate ligation where possible. The modelling is based on the Mn-anomalous diffraction data of Ferreira et al. (2004), which seem to be the most reliable at present and are available via the PDB database. Although these anomalous diffraction data are at $3.8 \AA$, they have the advantage of not being biased to the model phases and at this resolution is probably not influenced significantly by any radiation modifications. The models presented in the following sections have been fitted by real-space refinement against the $\mathrm{Mn}$-anomalous diffraction using the molecular graphics program, Соот (Emsley \& Cowtan 2004). Their validity will ultimately have to be reconciled with native electron density maps and with new structural data at higher resolution.

\section{(a) Modification of the Ferreira et al. model}

Our first approach was to modify the model of Ferreira et al. (2004) by recognizing that although EXAFS spectroscopy conducted by the Berkeley group ${ }^{1}$ indicates that there are three $\mathrm{Mn}$-di- $\mu$-oxo bridges but only one mono- $\mu$-oxo bridge (Yachandra 2002; Yano et al. 2006). Therefore, one possibility that we consider here to account for the discrepancy with the original Ferreira et al. model is to link Mn4 with the $\mathrm{Mn}_{3} \mathrm{Ca}^{2+} \mathrm{O}_{4}$ cluster via one of the $\mathrm{Mn}$ ions of the cubane (e.g. Mn3). Keeping the approximate positioning of $\mathrm{Ca}^{2+}$ proposed by Ferreira et al. (2004), confirmed by Loll et al. (2005) and recent work of Kargul et al. (2007), then the new bonding state for Mn4 leads to a repositioning of the other three $\mathrm{Mn}$ ions when compared with those in the model of Ferreira et al. (2001). This repositioning of the Mn ions therefore changes the protein ligation pattern as shown by comparing figure $1 c$ with figure $1 a$. The positioning of the amino acids has taken into account the possibility of bidentate bridging, which involved making slight changes in the side-chain positioning within the native electron density maps derived by Ferreira et al. (2004) and Loll et al. (2005). In this model, the same amino acids identified as part of the OEC by Ferreira et al. remain as metal ligands but the pattern is different: D1-His332 is a ligand to Mn1; D1-Asp342 can now form a bidendate bridging ligation with $\mathrm{Mn} 1$ and $\mathrm{Mn} 2$; and CP43-Glu354 also seems to be positioned so as to form a bidentate bridge between Mn2 and Mn3. A repositioning of the side chain of D1-Glu333, in agreement with Loll et al. (2005), suggests that its carboxylate could form a bridge between $\mathrm{Mn} 4$ and $\mathrm{Mn} 3$. As mentioned above, the Ferreira et al. positioning of D1-Asp170 is different from the Loll et al. structure. Assuming the Ferreira et al. positioning, D1-Asp 170, clearly makes a ligand for Mn4, and with a slight rotation of the carboxylate bond within the native electron density suggests that both oxygens could ligate to Mn4. The positioning of the side chain of D1-Glu189 suggests a monodentate ligation to $\mathrm{Mn} 1$, while reinterpreting the electron density of the C-terminal region of either the map of Ferreira et al. or Loll et al. is more difficult. It is clear that both
D1-Ala344 and D1-Asp342 are located close to Mn2, and with slight adjustment of their side chains in either map could mean that the oxygens of the carboxyl group of D1-Ala344 form a bridge between $\mathrm{Mn} 2$ and $\mathrm{Ca}^{2+}$ while D1-Asp342 forms a bidentate bridging ligand with $\mathrm{Mn} 1$ and $\mathrm{Mn} 2$. Figure $1 d$ shows a schematic of the ligation pattern for this model. The coordination number for $\mathrm{Mn}$ is 5 or 6 and, according to the model shown in figure $1 c$, this requirement is better satisfied by amino acid side chains than in the Ferreira et al. (2004) model due to the introduction of bidentate ligation: 6 for $\mathrm{Mn} 1 ; 6$ for $\mathrm{Mn} 2 ; 6$ for $\mathrm{Mn} 3$; and 4 for Mn4. The overall positioning of the $\mathrm{Mn}$ ions in this model and the pattern of amino acid ligation are similar to that proposed by Loll et al. (2005; with some exceptions). However, the average orientation to the membrane normal of the three $\mathrm{Mn}$-di- $\mu$-oxo-Mn bonds $\left(81^{\circ}\right)$ and the Mn-mono- $\mu$-oxo-Mn bond $\left(47^{\circ}\right)$ are not consistent with those recently derived from polarized EXAFS (Yano et al. 2006; Pushkar et al. 2007 ), which indicate an orientation of $40^{\circ}-60^{\circ}$ and $90^{\circ}$, respectively.

Although the major difference between the modified model and that of Ferreira et al. is the relocation of the $\mathrm{Mn}$ ions so as to accommodate only one $\mathrm{Mn}-$ mono- $\mu$ oxo-Mn bond, the slight changes in the orientation of some side chains also make some differences to the original Ferreira et al. model: D1-Ala344 is now in ligating distance to $\mathrm{Mn} 2$ as well as $\mathrm{Ca}^{2+}$ and bridging ligands are also proposed between $\mathrm{Mn} 1$ and Mn2 (D1-Asp342), Mn2 and Mn3 (CP43-Glu354) and between Mn3 and Mn4 (D1-Glu333).

\section{(b) EXAFS-based models}

The cubane model proposed by Ferreira et al. (figure $1 a$ ) or its modified form (figure 1c), or the Loll et al. model (2005), is not consistent with any of the four models recently proposed from polarized EXAFS spectroscopy as applied to single crystals (Yano et al. 2006). Therefore, we have investigated the EXAFS models using the $\mathrm{Mn}$-anomalous diffraction difference map derived by Ferreira et al. (2004). We found that none of the recently proposed EXAFS models fitted comfortably into the $\mathrm{Mn}$-anomalous diffraction map using the coordinates provided by Yano et al. (2006). Using real-space refinement against the anomalous $\mathrm{Mn}$ difference density to obtain a revised fitting and recognizing that there are discrepancies in the determination of the bond angles relative to the membrane normal, as emphasized by the comparison of Yano et al. (2006) with Pushkar et al. (2007) as well as with earlier work (George et al. 1989; Dittmer \& Dau 1998; Schiller et al. 1998), we slightly adjusted the positioning of the four EXAFS models so as to improve their fit within the Mn-anomalous difference map (figure 2). These new fittings within the $\mathrm{Mn}$-anomalous difference map give orientations to the membrane normal of the $\mathrm{Mn}$-di- $\mu$-oxo-Mn bonds (2.7 and $2.8 \AA$ ) and the $\mathrm{Mn}-$ mono- $\mu$-oxo-Mn bonds $(3.3 \AA$ ) as presented in table 1 , where they are compared with those derived from the coordinates of Yano et al. (2006). The differences are in most cases not large and probably within experimental error. 
Table 1. Calculated bond angles relative to the membrane normal for various models of the $\mathrm{Mn}_{4} \mathrm{Ca}^{2+}$ cluster of the OEC. (PDB coordinates 1S5L and 2AXT are for Ferreira et al. (2004) and Loll et al. (2005), respectively. Mono- $\mu$-cubane is the modified form of the Ferreira et al. model referred to model 1 in the text. Models I, II, IIa and III are the EXAFS-derived models of Yano et al. (2006), where the first values are calculated from the original orientations and the second after real-space fitting against the Mn-anomalous difference map of Ferreira et al. (2004). Last column is the angle of real-space rotation compared with the original of Yano et al. (2006).)

\begin{tabular}{|c|c|c|c|c|c|c|}
\hline structure & mono- $\mu$ & mean di- $\mu$ & $\begin{array}{l}\text { mean } 3.4 \AA \\
\text { (or nearer } \mathrm{Ca}^{2+} \\
\text { distances) }\end{array}$ & $\begin{array}{l}2.8 \AA \text { distance } \\
\text { angles }\end{array}$ & $\begin{array}{l}\text { mean } 2.7 \AA \\
\text { distance } \\
\text { angles }\end{array}$ & $\begin{array}{l}\text { rotation angle } \\
\text { (from Yano to } \\
\text { real-space fit) }\end{array}$ \\
\hline $1 \mathrm{~S} 5 \mathrm{~L}$ & $58^{\circ} / 37^{\circ}(2$ vals $)$ & $72^{\circ}$ & $39^{\circ}$ & & & n.a. \\
\hline $2 \mathrm{AXT}$ & $47^{\circ} / 84^{\circ}(2$ vals $)$ & $74^{\circ}$ & $31^{\circ}$ & & & n.a. \\
\hline mono- $\mu$-cubane & $47^{\circ}$ & $81^{\circ}$ & $29^{\circ}$ & & & n.a. \\
\hline model I & $34^{\circ}, 66^{\circ}$ & $61^{\circ}, 58^{\circ}$ & $61^{\circ}, 32^{\circ}$ & $85^{\circ}, 67^{\circ}$ & $49^{\circ}, 54^{\circ}$ & $34^{\circ}$ \\
\hline model II & $81^{\circ}, 87^{\circ}$ & $48^{\circ}, 55^{\circ}$ & $46^{\circ}, 33^{\circ}$ & $60^{\circ}, 65^{\circ}$ & $42^{\circ}, 50^{\circ}$ & $27^{\circ}$ \\
\hline model IIa & $86^{\circ}, 89^{\circ}$ & $50^{\circ}, 55^{\circ}$ & $37^{\circ}, 32^{\circ}$ & $57^{\circ}, 60^{\circ}$ & $46^{\circ}, 52^{\circ}$ & $36^{\circ}$ \\
\hline model III & $80^{\circ}, 83^{\circ}$ & $49^{\circ}, 52^{\circ}$ & $43^{\circ}, 36^{\circ}$ & $63^{\circ}, 65^{\circ}$ & $41^{\circ}, 46^{\circ}$ & $24^{\circ}$ \\
\hline
\end{tabular}

As shown in figure 2, the repositioning of the four EXAFS models into the crystal structure reveals the differences in possible amino acid ligation patterns for each model, which are also different from those indicated by Yano et al. (2006). As for the modified Ferreira et al. model in figure $1 c$, the orientations of the carboxylate side chains were optimized using the native electron density maps of Ferreira et al. (2004) and Loll et al. (2005), with the view of possibly forming bridging ligands whenever possible. All the amino acids identified by Ferreira et al. (2004) as potential ligands (with the exception of D1-Ala344 and D1-Asp342 of model I in figure $2 a, b$ ) remain the same but the precise arrangements are different. For EXAFS models II, IIa and III, D1-Ala344 forms a bidendate ligand with $\mathrm{MnD}$ (Mn1), while D1-Asp342 forms a monodentate ligand to this $\mathrm{Mn}$ (figure $2 d, f, h$ ). D1-His 332 remains a ligand for all four EXAFS models except for model I, where it ligates to $\mathrm{MnD}(\mathrm{Mn} 1)$, while for the other models it is a ligand for MnC (Mn2). D1-Glu333 is a ligand for MnA (Mn4) in all cases but with bidentate bridging to $\mathrm{MnB}(\mathrm{Mn} 3)$ in models II, IIa and III. In all cases, D1-Asp170 provides a single carboxylate ligand to MnA (Mn4). Only with EXAFS models I and III does D1-Glu189 form an exclusive bidentate ligand to $\mathrm{Ca}^{2+}$ while in the other two models it forms a bidentate bridge between $\mathrm{Ca}^{2+}$ and $\mathrm{MnC}(\mathrm{Mn} 2)$. The final amino acid ligand originally identified by Ferreira $e t a l$. (2004) is CP43-Glu354. This residue appears to be in ligating distance to $\mathrm{Mn}$ in all four EXAFS-based models. In model I, it is bidentate to $\mathrm{MnB}(\mathrm{Mn} 3$ ) while in model III its carboxylate oxygens bridge between $\mathrm{MnB}(\mathrm{Mn} 3)$ and $\mathrm{MnC}(\mathrm{Mn} 2)$. In models II and IIa, CP43-Glu354 provides a single ligand to $\mathrm{MnB}(\mathrm{Mn} 3)$.

\section{CONCLUSION}

In less than a decade, electron microscopy and X-ray crystallography have provided detailed information about the structure of PSII from both plants and cyanobacteria. The location and arrangement of the majority of the protein subunits of PSII have been revealed, including the organization of their transmembrane helices. The assignment of amino acids in these proteins as well as the organization of cofactors has been achieved by the application of X-ray crystallography of PSII isolated from cyanobacteria. Although the resolution has to date been restricted to 3.5-3.0 $\AA$, the modelling of side-chain positions is sufficiently good that there will only be slight modifications made to their positions as the resolution of the $\mathrm{X}$-ray diffraction data improves. Similarly, this will be true for most of the cofactors. For this reason, the current description of the protein environments around the various cofactors involved in energy transfer and charge separation is reasonably reliable. The only major uncertainties are the precise structure of the $\mathrm{Mn}_{4} \mathrm{Ca}^{2+}$ cluster and the nature of its protein surroundings in various S-state configurations, details which are paramount for elucidating the chemistry of the water-splitting reaction.

At present, the crystal structures of PSII are not at sufficient resolution to resolve the individual metal cations or the presence of low molecular weight ligands of the OEC. The situation appears to be further complicated by the possibility that radiation damage occurs at the metal cluster during the collection of $\mathrm{X}$-ray diffraction data. On the other hand, information about the structure of the $\mathrm{Mn}_{4} \mathrm{Ca}^{2+}$ cluster can be obtained at high spatial resolution and without radiation damage by EXAFS. Recent EXAFS studies have not only confirmed earlier determinations on $\mathrm{Mn}-\mathrm{Mn}$ and $\mathrm{Mn}-\mathrm{Ca}^{2+}$ distances but also imposed angular constraints for the metal-metal bonds relative to the membrane normal (Yano et al. 2006; Pushkar et al. 2007), although there are inconsistencies between these studies and with earlier studies by others (George et al. 1989; Dittmer \& Dau 1998; Schiller et al. 1998). Taken at face value, the most recent EXAFS work is not compatible with the models proposed by Ferreira et al. (2004) and Loll et al. (2005). Our modified version of the Ferreira et al. model presented in figure $1 c$ with one mono- $\mu$-oxo bond for the Mn4 linkage to the $\mathrm{Mn}_{3} \mathrm{Ca}^{2+} \mathrm{O}_{4}$ cluster also does not comply with the angular constraints imposed by the recent EXAFS analyses (table 1). The models derived from EXAFS studies of Yano et al. (2006) could therefore be considered to give a more realistic arrangement of the metal ions in the cluster, at least for the $S_{1}$ state, and perhaps one of the new positioning within the crystal structure reported here (figure 2) provides a better 
overall working model. However, this modelling could be criticized since it relies on X-ray diffraction data, which was suggested by Yano et al. (2005) not to be reliable for the OEC owing to radiation damage. But the $3.8 \AA \mathrm{Mn}$-anomalous difference data of Ferreira et al. (2004) were collected at low temperature and the density was consistent with the presence of four $\mathrm{Mn}$ ions, and therefore, at this low resolution, should be sufficiently reliable for inserting the EXAFS models even if some minor structural reorganization occurred due to radiation-induced reduction on $\mathrm{Mn}$ ions. Indeed, the four models we derived using the EXAFS models of Yano et al. (2006) and the real-space refinement against the $\mathrm{Mn}$-anomalous difference map of Ferreira et al. (2004) more or less maintained the angular properties for the various bond distances reported by Yano et al. (2006) (table 1). The possible protein ligation patterns for the four different EXAFSderived models are shown in figure 2 with only models II and IIa having the same pattern. The side-chain positioning has been modified slightly from the original modelling of Ferreira et al. (2004) by comparison with the electron density map of Ferreira et al. (2004) and Loll et al. (2005) and biasing to bidentate ligation and cross bridging whenever possible.

In conclusion, our analyses of the currently available structural data provide a number of alternative models for the metal-protein arrangements within the OEC. Improvement in the quality of X-ray diffraction data, collected in such a way as to reduce radiation damage, will be paramount for identifying which of these various models is the most reliable. Moreover, the resolution of $\mathrm{X}$-ray diffraction data must be such that it should clearly assign water molecules with the view of identifying those which act as the substrate during the catalytic cycle. This challenge, coupled with information derived from EXAFS, Fourier transform infrared (FTIR), electron paramagnetic (EPR) spectroscopies and other techniques, will ultimately provide the details required for understanding precisely how photosynthetic organisms use sunlight to split water.

We thank the Biotechnology and Biological Science Research Council for their financial support.

\section{ENDNOTE}

${ }^{1}$ There remains controversy in the literature regarding the number of $\mathrm{Mn}-$ mono- $\mu$-oxo-Mn and $\mathrm{Mn}$-di- $\mu$-oxo-Mn bonds based on EXAFS measurements. For example, Dau and colleagues provide evidence for two, not three, $\mathrm{Mn}$-di- $\mu$-oxo-Mn bonds in the $S_{1}$ state (Dau et al. 2004; Haumann et al. 2005).

\section{REFERENCES}

Archer, M. D. \& Barber, J. 2004 Photosynthesis and photoconversion. In Molecular to global photosynthesis, vol. II (eds M. D. Archer \& J. Barber) Photoconversion of solar energy, pp. 1-42. London, UK: Imperial College Press.

Barber, J. 2002 Photosystem II: a multisubunit membrane protein that oxidises water. Curr. Opin. Struct. Biol. 12, 523-530. (doi:10.1016/S0959-440X(02)00357-3)

Barber, J. 2003 Photosystem II: the engine of life. Biophys. $Q$. Rev. 36, 71-89. (doi:10.1017/S0033583502003839)
Barber, J., Ferreira, K. N., Maghlaoui, K. \& Iwata, S. 2004 Structure of the oxygen evolving center of photosystem II and its mechanistic implications. Phys. Chem. Chem. Phys. 6, 4737-4742. (doi:10.1039/b407981g)

Biesiadka, J., Loll, B., Kern, J., Irrgang, K. D. \& Zouni, A. 2004 Crystal structure of the cyanobacterial photosystem II at $3.2 \AA$ resolution: a closer look at the Mn-cluster. Phys. Chem. Chem. Phys. 6, 4733-4736. (doi:10.1039/ b406989g)

Christou, G. 1989 Manganese carboxylate chemistry and its biological relevance. Acc. Chem. Res. 22, 328-335. (doi:10.1021/ar00165a006)

Dau, H., Liebisch, P. \& Haumann, M. 2004 The structure of the manganese complex of photosystem II in its darkstable S1-state: EXAFS results in relation to recent crystallographic data. Phys. Chem. Chem. Phys. 6, 4781-4792. (doi:10.1039/b408146c)

Debus, R. J. 2001 Amino acid residues that modulate the properties of tyrosine $\mathrm{Y}-\mathrm{Z}$ and the manganese cluster in the water oxidizing complex of photosystem II. Biochim. Biophys. Acta 1503, 164-186. (doi:10.1016/S00052728(00)00221-8)

Diner, B. A. 2001 Amino acid residues involved in the coordination and assembly of the manganese cluster of photosystem II. Proton-coupled electron transport of the redox-active tyrosines and its relationship to water oxidation. Biochim. Biophys. Acta 1503, 147-163. (doi:10.1016/S0005-2728(00)00220-6)

Diner, B. A. \& Babcock, G. T. 1996 Structure, dynamics and energy conversion efficiency in photosystem II. In Advances in photosynthesis, (eds D. R. Ort \& C. F. Yocum) Oxygenic photosynthesis: the light reactions, pp. 213247. Dordrecht, The Netherlands: Kluwer Academic Publisher.

Diner, B. A., Nixon, P. \& Farchaus, J. W. 1991 Site-directed mutagenesis of photosynthetic reaction centers. Curr. Opin. Struct. Biol. 1, 546-554. (doi:10.1016/S0959440X(05)80076-4)

Dismukes, G. C. 1996 Manganese enzymes with binuclear active sites. Chem. Rev. 96, 2909-2926. (doi:10.1021/ cr950053c)

Dittmer, J. \& Dau, H. 1998 Theory of the linear dichroism in the extended X-ray absorption fine structure (EXAFS) of partially vectorially ordered systems. F. Phys. Chem. B 102, 8196-8200. (doi:10.1021/jp981049i)

Emsley, P. \& Cowtan, K. 2004 Cоот: model-building for molecular graphics. Acta Cryst. Sec. D Biol. Cryst. 60, 2126-2132. (doi:10.1107/S0907444904019158)

Ferreira, K. N., Iverson, T. M., Maghlaoui, K., Barber, J. \& Iwata, S. 2004 Architecture of the photosynthetic oxygenevolving center. Science 303, 1831-1838. (doi:10.1126/ science.1093087)

George, G. N., Prince, R. C. \& Cramer, S. P. 1989 The manganese site of the photosynthetic water-splitting enzyme. Science 243, 789-791. (doi:10.1126/science. 2916124)

Grabolle, M., Haumann, M., Muller, C., Liebisch, P. \& Dau, H. 2006 Rapid loss of structural motifs in the manganese complex of oxygenic photosynthesis by X-ray irradiation at $10-300 \mathrm{~K}$. F. Biol. Chem. 281, 4580-4588. (doi:10. 1074/jbc.M509724200)

Hasegawa, K., Ono, T. A., Inoue, Y. \& Kusunoki, M. 1999 Spin-exchange interactions in the S2-state manganese tetramer in photosynthetic oxygen-evolving complex deduced from $g=2$ multiline EPR signal. Chem. Phys. Lett. 300, 9-19. (doi:10.1016/S0009-2614(98)01369-4)

Haumann, M., Muller, C., Liebisch, P., Iuzzolino, L., Dittmer, J., Grabolle, M., Neisius, T., Meyer-Klaucke, W. \& Dau, H. 2005 Structural and oxidation state changes of the photosystem II manganese complex in four 
transitions of the water oxidation cycle $(\mathrm{S} 0 \rightarrow \mathrm{S} 1, \mathrm{~S} 1 \rightarrow \mathrm{S} 2$, $\mathrm{S} 2 \rightarrow \mathrm{S} 3$, and $\mathrm{S} 3,4 \rightarrow \mathrm{S} 0$ ) characterized by X-ray absorption spectroscopy at $20 \mathrm{~K}$ and room temperature. Biochemistry 44, 1894-1908. (doi:10.1021/bi048697e)

Hoganson, C. W. \& Babcock, G. T. 1997 A metalloradical mechanism for the generation of oxygen from water in photosynthesis. Science 277, 1953-1956. (doi:10.1126/ science.277.5334.1953)

Kamiya, N. \& Shen, J. R. 2003 Crystal structure of oxygenevolving photosystem II from Thermosynechococcus vulcanus at $3.7 \AA$ resolution. Proc. Natl Acad. Sci. USA 100, 98-103. (doi:10.1073/pnas.0135651100)

Kanyo, Z. F., Scolnick, L. R., Ash, D. E. \& Christianson, D. W. 1996 Structure of a unique binuclear manganese cluster in arginase. Nature 383, 554-557. (doi:10.1038/ 383554a0)

Kargul, J., Maghlaoui, K., Murray, J. W., Deak, Z., Boussac, A., Rutherford, A. W., Vass, I. \& Barber, J. 2007 Purification, crystallization and X-ray diffraction analyses of the T. elongatus PSII core dimer with strontium replacing calcium in the oxygen-evolving complex. Biochim. Biophys. Acta 1767, 404-413.

Loll, B., Kern, J., Saenger, W., Zouni, A. \& Biesiadka, J. 2005 Towards complete cofactor arrangement in the $3.0 \AA$ resolution structure of photosystem II. Nature 438, 1040-1044. (doi:10.1038/nature04224)

Lundberg, M. \& Siegbahn, P. E. M. 2004 Theorectical investigations of the structure and mechanism of the oxygen-evolving complex in PSII. Phys. Chem. Chem. Phys. 6, 4772-4780. (doi:10.1039/b406552b)

McEvoy, J. P. \& Brudvig, G. W. 2004 Structure-based mechanism of photosynthetic water oxidation. Phys. Chem. Chem. Phys. 6, 4754-4763. (doi:10.1039/ b407500e)

McEvoy, J. P. \& Brudvig, G. W. 2006 Water-splitting chemistry of photosystem II. Chem. Rev. 106, 4455-4483. (doi:10.1021/cr0204294)

Misra, A., Wernsdorfer, W., Abboud, K. A. \& Christou, G. 2005 The first high oxidation state manganese-calcium cluster: relevance to the water oxidizing complex of photosynthesis. Chem. Commun. (Camb.) 1, 54-56. (doi:10.1039/b413680b)

Murray, J. W. \& Barber, J. 2006 Identification of a calciumbinding site in the PsbO protein of photosystem II. Biochemistry 45, 4128-4130. (doi:10.1021/bi052503t)

Murray, J. W. \& Barber, J. 2007 Structural characteristics of channels and pathways in photosystem II including the identification of an oxygen channel. F. Struct. Biol. 159, 228-237. (doi:10.1016/j.jsb.2007.01.016)

Peloquin, J. M., Campbell, K. A., Randall, D. W., Evanchik, M. A., Pecoraro, V. L., Armstrong, W. H. \& Britt, R. D. $200055 \mathrm{Mn}$ ENDOR of the S2-state multiline EPR signal of photosystem II: implications on the structure of the tetranuclear $\mathrm{Mn}$ cluster. f. Am. Chem. Soc. 122, 10 926-10 942. (doi:10.1021/ja002104f)

Pushkar, Y., Yano, J., Glatzel, P., Messinger, J., Lewis, A., Saure, K., Bergmann, U. \& Yachandra, V. K. 2007 Structure and orientation of the $\mathrm{Mn}_{4} \mathrm{Ca}^{2+}$ cluster in plant photosystem II membranes studied by polarized range-extended X-ray absorption spectroscopy. F. Biol. Chem. 282, 7198-7208. (doi:10.1074/jbc.M610505200)

Schiller, H., Dittmer, J., Iuzzolino, L., Dörner, W., MeyerKlaucke, W., Sole, V. A., Nolting, H.-F. \& Dau, H. 1998 Structure and orientation of the oxygen-evolving manganese complex of green algae and higher plants investigated by X-ray absorption linear dichroism spectroscopy on oriented photosystem II membrane particles. Biochemistry 37, 7340-7350. (doi:10.1021/bi972329b)
Siegbahn, P. E. M. 2006 O-O bond formation in the $\mathrm{S}_{4}$-state of the oxygen evolving complex in photosystem II. Chem. Eur. F. 12, 9217-9237. (doi:10.1002/chem.200600774)

Siegbahn, P. E. M. \& Lundberg, M. 2005 The mechanism for dioxygen formation in PSII studied by quantum chemical methods. Photochem. Photobiol. Sci. 4, 1035-1043. (doi:10.1039/b506746b)

Sommerhalter, M., Saleh, L., Bollinger Jr, J. M. \& Rosenzweig, A. C. 2005 Structure of Escherichia coli ribonucleotide reductase R2 in space group P6122. Acta Crystallogr. D: Biol. Crystallogr. 61, 1649-1654. (doi:10. 1107/S0907444905034062)

Sproviero, E. M., Gascon, J. A., McEvoy, J. P., Brudvig, G. W. \& Batista, V. S. 2006 QM/MM models of the $\mathrm{O}_{2^{-}}$ evolving complex of photosystem II. f. Chem. Theory Comput. 2, 1119-1134. (doi:10.1021/ct0600181)

Sproviero, E. M., Gascon, J. A., McEvoy, J. P., Brudvig, G. W. \& Batista, V. S. 2007 Quantum mechanics/molecular mechanics structural models of the oxygen-evolving complex of photosystem II. Curr. Opin. Struct. Biol. 17, 173-180. (doi:10.1016/j.sbi.2007.03.015)

Wydrzynski, T. J. \& Satoh, K. 2005 Photosystem II. The light-driven water:plastoquinone oxidoreductase. Advances in photosynthesis and respiration, vol. 22, pp. 1-786. Dordrecht, The Netherlands: Springer.

Yachandra, V. K. 2002 Structure of the Mn complex in photosystem II: insights from X-ray spectroscopy. Phil. Trans. R. Soc. B 357, 1347-1357. (doi:10.1098/rstb. 2002.1133)

Yano, J. et al. $2005 \mathrm{X}$-ray damage to the $\mathrm{Mn}_{4} \mathrm{Ca}$ complex in photosystem II crystals: a case study for metallo-protein X-ray crystallography. Proc. Natl Acad. Sci. USA 102, 12 047-12 052. (doi:10.1073/pnas.0505207102)

Yano, J. et al. 2006 Where water is oxidised to dioxygen: structure of the photosynthetic $\mathrm{Mn}_{4} \mathrm{Ca}^{2+}$ cluster. Science 314, 821-825. (doi:10.1126/science.1128186)

Zouni, A., Witt, H. T., Kern, J., Fromme, P., Krauss, N., Saenger, W. \& Orth, P. 2001 Crystal structure of photosystem II from Synechococcus elongatus at $3.8 \AA$ resolution. Nature 409, 739-743. (doi:10.1038/ 35055589)

\section{Discussion}

V. L. Pecoraro (University of Michigan, Michigan, USA). What is the $\mathrm{Ca}^{2+}-\mathrm{Mn}$ (dangler) distance in your various models?

J. Barber. In all the models, this distance varies between 4 and $5 \AA$, which is similar to the distance between $\mathrm{Fe}$ and $\mathrm{Cu}$ in the catalytic centre of cytochrome oxidase. H. Dau (Freie University, Berlin, Germany). We found that bromide (in bromide-repleted PSII) is not a firstsphere ligand for the metal cluster but possibly at a distance of approximately $5.5 \AA$ to $\mathrm{Mn}$ or $\mathrm{Ca}$ ions. Could that distance be in agreement with your crystallographic data?

J. Barber. After infiltrating our PSII crystals with bromide, we detected two binding sites based on anomalous diffraction, which were approximately $7 \AA$ away from $\mathrm{Mn}$ and would therefore agree with you. But it is possible that we did not exchange with a high affinity site for halide binding which could be in the ligation sphere of the $\mathrm{Mn}_{4} \mathrm{Ca}^{2+}$ cluster. We need to repeat the experiment on PSII isolated from cells grown in bromide.

G. Brudvig (Yale University, Yale, USA). You emphasized bidentate coordination of carboxylate ligands. It 
is known from iron metalloproteins that carboxylates favour monodentate terminal coordination for higher oxidation states, in what is known as the 'carboxylate shift' when iron is oxidized from $\mathrm{Fe}$ (II) to $\mathrm{Fe}$ (III). I would expect carboxylates to favour terminal ligation to $\mathrm{Mn}$ in the OEC so that the negative charge can better stabilize the high oxidation state. How many bidentate bridging carboxylates are in your latest models?

J. Barber. I have presented five different models, one being a modification of the original Ferreira et al. (2004) structure (figure 1 above) and four based on combining the crystal structure data of Ferreira et al. and the recent four models derived from EXAFS by Yano et al. (2006). These are shown in figures 1 and 2, respectively, in the paper above where it can be seen that the number of bidentate bridging ligands varies for each model.

P. Siegbahn (Stockholm University, Stockholm, Sweden). Bidentate ligation of the carboxylates in the OEC is in general a good idea between different metals since there are so few amino acid ligands. However, having D1Asp170 bidentate to one single metal ion (Mn4) is very unlikely in my opinion.

J. Messinger (Muelheim, Germany). Jim, during the construction of your new models for the OEC, you appear to rotate our EXAFS model of the $\mathrm{Mn}_{4} \mathrm{O}_{x} \mathrm{Ca}$ cluster (Yano et al. 2006) within your electron density in order to achieve a better fit for the ligands. I like to point out that the polarized EXAFS data on PSII crystals also define the orientation of the $\mathrm{Mn}_{4} \mathrm{O}_{x} \mathrm{Ca}$ cluster within
PSII, and that therefore a rotation leads to significant discrepancy between calculated and measure single crystal EXAFS spectra. The only freedom you have are translations parallel to the $\mathrm{a}, \mathrm{b}$ and $\mathrm{c}$ axes. As discussed in Yano et al. (2006), more likely reasons for the unsatisfactory positions of many ligands in the models presented in Yano et al. (2006) are (i) the limited resolution of the X-ray crystallography (XRD) measurements and (ii) ligand movements during crystallography caused by photoreduction and disruption of the $\mathrm{Mn}_{4} \mathrm{O}_{x-}$ Ca cluster during XRD data collection (Yano et al. 2005). A more promising approach for finding a reliable highresolution structure of the OEC (i.e. of both ligands and $\mathrm{Mn}_{4} \mathrm{O}_{x} \mathrm{Ca}$ cluster) appears to be DFT calculations that optimize the ligand positions while the metal ions are fixed in place. Such models need of course be further evaluated by DFT-based predictions of spectroscopic parameters. Would you agree with these comments?

J. Barber. I absolutely agree that DFT and QM/MM calculations of the type presented at this meeting by Per Siegbahn, Victor Batista and yourself are very important indeed to test the validity of proposed models either derived from crystallography or EXAFS. However, EXAFS gives no information about protein ligands and therefore we are forced to blend X-ray diffraction and spectroscopy data. Our attempts to do this, together with their short comings are described in our paper (see text and figure 2), and the changes in orientations of the EXAFS models are within the experimental errors as shown in table 1 . 